\title{
MORSE THEORY FOR CODIMENSION-ONE FOLIATIONS
}

\author{
STEVEN C. FERRY AND ARTHUR G. WASSERMAN
}

\begin{abstract}
It is shown that a smooth codimension-one foliation on a compact simply-connected manifold has a compact leaf if and only if every smooth realvalued function on the manifold has a cusp singularity.
\end{abstract}

Introduction. Morse theory is a method of obtaining information about a smooth manifold by analyzing the singularities of a generic real-valued function on the manifold. If the manifold has the additional structure of a foliation, one can then consider as well the singularities of the function restricted to each leaf. The resulting singular set becomes more complicated, higher dimensional, and less trivially placed. In exchange, one obtains information about the foliation.

In this paper we consider codimension-one smooth foliations and show that, in this case, the occurrence of one type of singularity, the cusp, is related to the existence of compact leaves. Specifically, we show that a smooth codimension-one foliation on a compact simply-connected manifold has a compact leaf if and only if every real-valued function on $M$ has a cusp.

The singularities of a function on a foliated manifold are very similar to the singularities of a map to the plane. See $\left[\mathbf{F}, \mathbf{H}-\mathbf{W}, \mathbf{L}_{\mathbf{1}}, \mathbf{T}_{\mathbf{1}}, \mathbf{W}_{\mathbf{a}}, \mathbf{W}\right]$. However, Levine $\left[\mathbf{L}_{1}\right]$ has shown that the only obstruction to finding a map of a compact manifold to the plane without a cusp singularity is the $\bmod 2$ Euler characteristic of the manifold.

Similarly, one can consider the singularities of a smooth (not necessarily closed) 1 -form on a foliated manifold. Compact leaves are not an obstruction to finding a 1 -form without cusp singularity in this case either, as one can easily see by considering any foliation of $S^{3}$.

The organization of the paper is as follows: $\$ 1$ contains the basic definitions as well as canonical examples of the singularities of a generic Morse function on a foliated manifold; $\$ 2$ has the statement of results and derives the main theorem of the paper, Corollary 2.2 from the cancellation theorem and Novikov's theory of components [N]; $\$ 3$ proves a two-dimensional version of the cancellation theorem; $\$ 4$ completes the proof of the cancellation theorem.

Received by the editors November 4, 1985.

1980 Mathematics Subject Classification (1985 Revision). Primary 57R30, 53C12.

Key words and phrases. Codimension-one foliation, compact leaf, Morse theory, cusp singularity.

C1986 American Mathematical Society $0002-9947 / 86 \$ 1.00+\$ .25$ per page 


\section{Preliminaries.}

Definition. A smooth codimension-one foliation, $\mathscr{F}$, of a manifold $M^{n}$ is a collection $\left\{\left(U_{i}, h_{i}\right)\right\}_{i \in I}$ of open sets $U_{i} \subset M$ and smooth diffeomorphisms $h_{i}$ : $U_{i} \rightarrow \mathbf{R}^{n}$ such that

(i) $\cup_{i \in I} U_{i}=M$,

(ii) if $U_{i} \cap U_{j} \neq \varnothing$, then the map $\gamma_{i j}=h_{i} \circ h_{j}^{-1}: h_{j}\left(U_{i} \cap U_{j}\right) \rightarrow h_{i}\left(U_{i} \cap U_{j}\right)$ preserves vertical $(n-1)$ planes. That is, if we write

$$
\gamma_{i j}\left(X^{1}, \ldots, X^{n}\right)=\left(\gamma_{i j}^{1}\left(X^{1}, \ldots, X^{n}\right), \ldots, \gamma_{i j}^{n}\left(X^{1}, \ldots, X^{n}\right)\right)
$$

then $\gamma_{i j}^{1}$ is a function of $X^{1}$ only.

(iii) The collection $\left\{\left(U_{i}, h_{i}\right)\right\}_{i \in I}$ is maximal with respect to property (ii).

Intuitively, $\mathscr{F}$ decomposes $M$ into a union of $(n-1)$-dimensional manifolds (the leaves of the foliation) which fit together locally like $(n-1)$ planes in $\mathbf{R}^{n}$. We denote the leaf through the point $p \in M$ by $L(p)$. The maps $g_{i}=\pi_{i} \circ h_{i}$ where $\pi_{1}: \mathbf{R}^{n} \rightarrow \mathbf{R}$ is projection onto the first factor are called the distinguished submersions of $\mathscr{F}$.

The tangent bundle of $M, T(M)$, can be written as $T(M)=T(\mathscr{F}) \oplus \xi$ where $T(\mathscr{F})$ consists of vectors tangent to the leaves and $\xi$ is a line bundle transverse to the foliation. If $\xi$ is orientable, a choice of orientation for $\xi$ is called a transverse orientation of $F$ and $\mathscr{F}$ is called transversely oriented.

We will be studying real-valued functions on foliated manifolds and will need an analogue of the usual theory of singularities of differentiable maps from $M^{n}$ to $\mathbf{R}^{2}$. See [F, H, Ha, $\left.\mathbf{L}_{1}, \mathbf{L}_{\mathbf{2}}, \mathbf{T}_{\mathbf{1}}, \mathbf{T}_{\mathbf{2}}, \mathbf{W}_{\mathbf{a}}, \mathbf{W}\right]$. The analogy is this: locally, the foliation is given by the level sets of a submersion, $g$, of an open set $U$ of $M$ into $\mathbf{R}$; the singular set of $f$ on $(M, \mathscr{F})$ will be, locally, the singular set of the pair $(f \mid U, g)$. This is easily seen to be independent of the choice of $g$. Alternatively, if $\mathscr{F}$ is transversely orientable, we may define the foliation by a nonsingular one-form $w$ which is unique up to multiplication by a nonzero function. The singular set, $S$, is then $S=\{p \in$ $\left.M \mid w_{p} \wedge d f_{p}=0\right\}$.

Definition. A point $p \in M$ is an $\mathscr{F}$-regular point if $d(f \mid L(p))_{p} \neq 0$.

Note that $p$ is an $\mathscr{F}$-regular point for $f$ iff $p$ is a regular point of $f \mid L(p)$ or, equivalently, the leaf $L(p)$, meets the level set $f^{-1}(f(p))$ transversely at $p$.

If $p$ is not an $\mathscr{F}$-regular point we say that $p$ is a singular point of $f$ on $(M, \mathscr{F})$ and we let $S=S(f)$ denote the set of singular points.

We say that $S$ is in general position with respect to $\mathscr{F}$ if, for any distinguished submersion $g, g \mid S$ is a Morse function, i.e. has only nondegenerate critical points.

Let $C(M)$ denote the set of smooth real-valued functions on $M$ with the $C^{k}$ topology for some $k \geqslant 2$.

Proposition 1.1 [F]. There is an open, dense set $\mathcal{O} \subset C(M)$ such that for each $f \in \mathcal{O}$

(i) $S(f)$ is a submanifold of $M$ of dimension one and,

(ii) $S(f)$ is in general position with respect to $f$.

Henceforth we will only consider functions in $\mathcal{O}$.

There are three types of generic singularities of a real-valued function in $\mathcal{O}$. We will list them, name them, and give the canonical form for each. 
Let the points of $\mathbf{R}^{n}$ be denoted by $(x, y, Z)$ where $x, y \in \mathbf{R}$ and $Z \in \mathbf{R}^{n-2}$, let $\mathbf{R}^{n}$ be foliated by the planes $x=$ constant, and let the vector field $\partial / \partial x$ determine a transverse orientation for this foliation. Our canonical forms are achieved by leaf-preserving diffeomorphisms which respect the transverse orientation of the foliation. If $n \geqslant 3$, these diffeomorphisms can be chosen to be orientation preserving as well.

The canonical form at an $\mathscr{F}$-regular point is given by $f(x, y, Z)=y$.

Definition. A point $p$ is a regular pseudocritical point if

(i) $d f_{p} \neq 0$,

(ii) $d(f \mid L(p))_{p}=0$ and,

(iii) $d^{2}(f \mid L(p))_{p}$ is nonsingular.

The canonical form for a regular pseudocritical point is $f(x, y, Z)= \pm x \pm y^{2}+$ $Q(Z)$ where $Q$ is a nonsingular quadratic form. Note that, in this case, the level set $f^{-1}(f(p))$ is a manifold tangent to $L(p)$ at $p$. See Figure 1a.

Definition. A point $p$ is an $\mathscr{F}$-critical point of $f$ if

(i) $d f_{p}=0$,

(ii) $d^{2} f_{p}$ is nonsingular,

(iii) $d^{2}(f \mid L(p))_{p}$ is nonsingular.

The canonical form for an $\mathscr{F}$-critical point is $f(x, y, Z)= \pm x^{2} \oplus y^{2}+Q(Z)$ where $Q$ is a nonsingular quadratic form. Note that conditions (ii) and (iii) are independent on $\mathbf{R}^{2}, f=x y$ satisfies (i) and (ii) but not (iii), $f=y^{2}$ satisfies (i) and (iii) but not (ii). At an $\mathscr{F}$-critical point $p$ the level set $f^{-1}(f(p))$ is not necessarily a manifold. See Figure 1b.

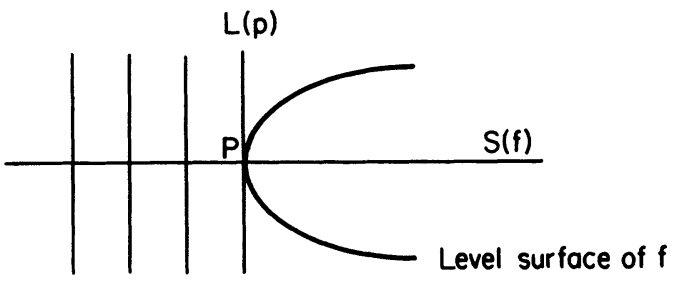

FIGURE 1a. The point $p$ is a regular pseudocritical point

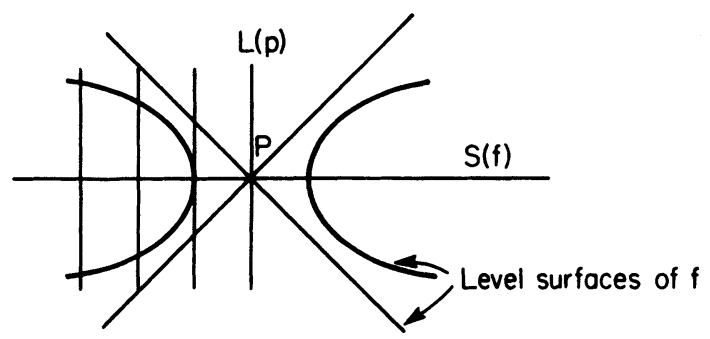

FIgURE $1 \mathrm{~b}$. The point $p$ is an $\mathscr{F}$-critical point 


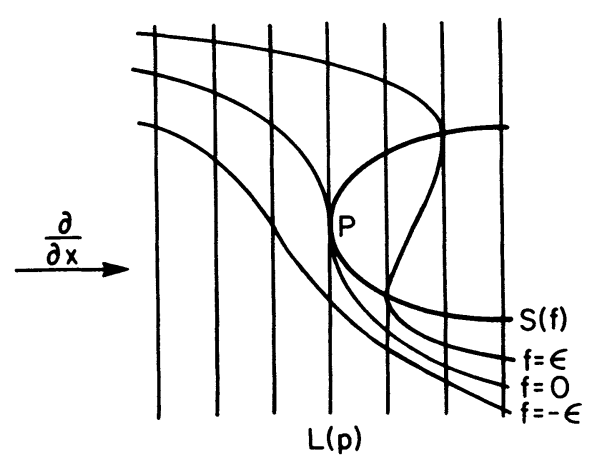

Figure 2a

The point $p$ is a negative turning point

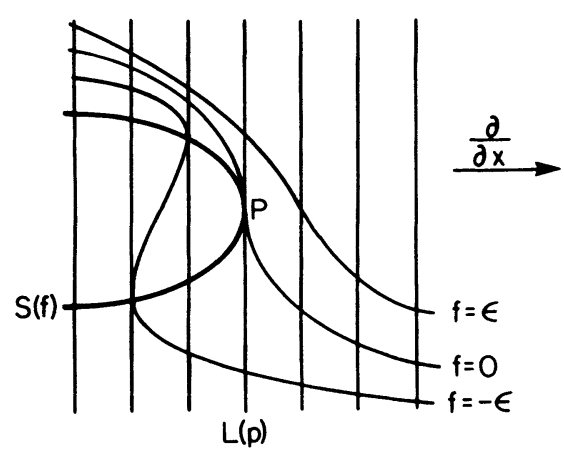

Figure $2 b$

The point $p$ is a positive turning point

Definition. A point $p$ is called a turning point of $f \in \mathcal{O}$ if

(i) $d f_{p} \neq 0$,

(ii) $d(f \mid L(p))_{p}=0$,

(iii) $g \mid S(f)$ has a critical point at $p$ for some distinguished submersion $g$,

(iv) $d_{p}^{2}(f \mid L(p))$ is singular of rank $n-2$ and,

(v) $d_{q}^{2}(f \mid L(q))$ is nonsingular for $q$ in a deleted neighborhood of $p$ in $S$.

The canonical form for a turning point is given by $f(x, y, Z)= \pm x \pm x y+\psi(y)$ $+Q(Z)$ where $Q$ is again a nonsingular quadratic form and $\psi$ is an odd function with $\psi^{\prime}(0)=0, \psi^{\prime \prime \prime}(0)>0$. The unusually complicated expression for $f$ is due to our restriction to leaf-preserving diffeomorphisms.

THEOREM $1.2[\mathbf{F}]$. There is an open dense set $\mathcal{O}^{\prime} \subset \mathcal{O}$ such that every $f \in \mathcal{O}^{\prime}$ has only the singularities listed above. Furthermore, any $f \in \mathcal{O}^{\prime}$ may be approximated $C^{0}$ by an $\tilde{f} \in \mathcal{O}^{\prime}$ such that at every turning point $p$ of $\tilde{f}$ we may take $\psi(y)=y^{3}$.

The regular pseudocritical points and the $\mathscr{F}$-critical points correspond to fold points and the turning points correspond to cusps under the analogy to maps to the plane.

Turning points having the form $f(x, y, z)= \pm x+x y+\psi(y)+Q(Z)$ are called positive, those with canonical form $f(x, y, Z)= \pm x-x y+\psi(y)+Q(Z)$ are called negative. See Figures $2 \mathrm{a}$ and $2 \mathrm{~b}$. (We require $\mathscr{F}$ to be transversely oriented.)

At every point $p \in S(f)$ we have the (possibly singular) quadratic form $d^{2}(f \mid L(p))$; the index of this form is called the leaf index of $p$. Note that the leaf index is constant along $S(f)$ except at turning points and at a turning point of leaf index $\lambda$ the leaf index changes from $\lambda$ to $\lambda+1$.

We now recall the definition of a Novikov component of a codimension-one foliation, $\mathscr{F}$, on a compact manifold $M$.

DEFINITION. We say that $x$ and $y$ in $M$ are equivalent and write $x \sim y$ if either

(i) $x$ and $y$ lie in the same leaf of $\mathscr{F}$ or

(ii) there is a closed transversal to the foliation which passes through $x$ and $y$.

THEOREM 1.3 (NOVIKOV [N]). (a) is an equivalence relation,

(b) if there is no closed transversal through $p$, then $L(p)$ is compact. 
DEFINITION. The equivalence classes defined above are called the Novikov components of $\mathscr{F}$.

TheOREM 1.4 (Novikov [N]). Every Novikov component is either a compact leaf or an open submanifold with boundary a finite union of compact leaves which are components.

2. Statement of results. Let $\mathscr{F}$ be a smooth codimension-one foliation on a compact-connected manifold $M^{n}, n \geqslant 3$. We then have the main theorem of the paper.

THEOREM 2.1. Statements (a), (b), and (c) below are equivalent

(a) $\mathscr{F}$ has only one Novikov component.

(b) There is a generic function $f:(M, \mathscr{F}) \rightarrow \mathbf{R}$ which has no turning points.

(c) There is a generic Morse function $f: M \rightarrow \mathbf{R}$ such that $f \mid L$ is a Morse function for every leaf $L$ of $\mathscr{F}$.

COROllary 2.2. If $H_{1}\left(M ; Z_{2}\right)=0$ then (a), (b), (c) are equivalent to

(d) $\mathscr{F}$ has no compact leaf.

Proof of Corollary 2.2. If $\mathscr{F}$ has no compact leaf then each Novikov component is open and hence, by connectedness, $\mathscr{F}$ has only one Novikov component. Thus (d) implies (a).

To see that (a) implies (d) we note that there must be a closed transversal through any point in $M$ if $\mathscr{F}$ has only one component. If $L$ is a compact leaf of $\mathscr{F}$, then $L$ separates $M$ because $H_{1}\left(M ; Z_{2}\right)=0$. Furthermore, $\mathscr{F}$ is transversely orientable because $w_{1}(\xi) \in H^{1}\left(M ; Z_{2}\right) \approx H_{1}\left(M ; Z_{2}\right)=0$. Thus the proof will be complete if we establish

LEMMA 2.3. There does not exist a closed transversal to a transversely orientable codimension-one foliation through any compact separating leaf.

Proof of Lemma 2.3. Choose a nonzero section $N$ of $\xi$ and a Riemannian metric for $M$. Let $w: I \rightarrow M$ be a closed transversal through the compact leaf $L$. Then $\left\langle w^{\prime}(t), N_{w(t)}\right\rangle \neq 0$ since $w$ is a transversal. If $M-L=A \cup B$ and if $N \mid L$ points into $A$ and $w\left(t_{1}\right), w\left(t_{2}\right) \in L, t_{1}<t_{2}$ and $w(t) \in A$, say, for $t_{1}<t<t_{2}$, then

$$
\left\langle w^{\prime}\left(t_{1}\right), N_{w\left(t_{1}\right)}\right\rangle>0 \text { and }\left\langle w^{\prime}\left(t_{2}\right), N_{w\left(t_{2}\right)}\right\rangle<0
$$

and hence $\left\langle w^{\prime}(t), N_{w(t)}\right\rangle=0$ for some $t$, contradicting the fact that $w$ is a transversely.

REMARK. It is easy to construct a codimension-one foliation $\mathscr{F}$ on the Klein bottle having a compact separating leaf $L$ such that there is a closed transversal through $L$. Of course, $\mathscr{F}$ is not transversely oriented.

Definition. A transverse arc, $w: I \rightarrow M$, to a transversely oriented codimensionone foliation $\mathscr{F}$ is positively oriented (resp. negatively oriented) if $\left\langle w^{\prime}(t), N_{w(t)}\right\rangle>0$ (resp. $\left\langle w^{\prime}(t), N_{w(t)}\right\rangle<0$ ) where $N$ is a positively oriented nonzero section of $\xi$ and $\langle$,$\rangle is any Riemannian metric.$

Our main tool in proving Theorem 2.1 is the following cancellation theorem. 
THEOREM 2.4. Let $\mathscr{F}$ be a smooth transversely oriented codimension-one foliation on the compact manifold $M$. Let $f:(M, \mathscr{F}) \rightarrow \mathbf{R}$ be a generic function and let $w: I \rightarrow M$ be a positively oriented transversal. Suppose $w(t)$ is an F्Fregular point of $f$ for $0<t<1, w(0)=p$ is a positive turning point of index $\lambda$, and $w(1)=q$ is a negative turning point of index $\lambda$. Let $U$ be any neighborhood of $w(I)$. Then there is a generic function $\bar{f}:(M, \mathscr{F}) \rightarrow \mathbf{R}$ such that $f \equiv \bar{f}$ outside of $U$ and $\bar{f}$ has two fewer turning points than $f$.

Proof. The proof of Theorem 2.4 will comprise $\$ \S 3$ and 4 of this paper.

We now show that Theorem 2.4 implies Theorem 2.1.

Proof of TheOrem 2.1. (c) $\Rightarrow$ (b). If $p$ is a turning point for $f$ then $d(f \mid L(p))_{p}$ $=0$ and $d_{p}^{2}(f \mid L(p))_{p}$ is singular and hence $p$ is a degenerate critical point of $f \mid L(p)$. Thus $f \mid L(p)$ is not a Morse function.

(b) $\Rightarrow$ (c). Follows as easily by looking at the canonical forms.

(b) $\Rightarrow$ (a). If $f$ has no turning point then $S(f)$ consists of closed transversals as can be seen by looking at the canonical forms for a regular pseudocritical point and an $\mathscr{F}$-critical point. If $\mathscr{F}$ has more than one component then there must be a compact leaf, $L_{0}$, which is in the boundary of a Novikov component and hence is itself a Novikov component. But $f \mid L_{0}$ has a maximum at $p_{0}$, say, and thus $d\left(f \mid L_{0}\right)_{p_{0}}=0$ so $L_{0} \cap S(f) \neq \varnothing$. Thus there is a closed transversal through $L_{0}$. This contradiction shows $\mathscr{F}$ can have only one Novikov component.

(a) $\Rightarrow(\mathrm{b})$. Let $f:(M, \mathscr{F}) \rightarrow \mathbf{R}$ be a generic function. Since the turning points of $f$ are isolated they are finite in number and it suffices, by induction, to show we can apply Theorem 2.4 to cancel a pair of turning points.

Let $p$ be a turning point of maximum leaf index $\lambda$; then the leaf index changes at $p$ from $\lambda$ to $\lambda+1$ and as we travel around this component of the singular set the leaf index must return to $\lambda$ i.e. there must be another turning point of index $\lambda, q$ say, and there is no intervening turning point by the maximality of $\lambda$. We show now that $p$ and $q$ can be cancelled.

Suppose that $\mathscr{F}$ is transversely oriented by a nonzero section $N$ of $\xi$. Let $\gamma$ : $I \rightarrow S(f)$ be a parameterization of the component of $S(f)$ containing $p$ and $q$. Note that at a positive turning point $\left\langle\gamma^{\prime}(t), N_{\gamma(t)}\right\rangle$ changes from positive to negative and at a negative turning point $\left\langle\gamma^{\prime}(t), N_{\gamma(t)}\right\rangle$ changes from negative to positive. Thus positive and negative turning points alternate and we may assume $p$ positive, $q$ negative. Finally, since $\mathscr{F}$ has only one component there is a positively oriented closed transversal $\tilde{w}: I \rightarrow M$ through $p$ and $q$ which we may assume, by general position, meets $S(f)$ in $p$ and $q$ only. Let $w$ denote the positively oriented transverse arc from $p$ to $q$ obtained by restricting $q$. This fulfills the conditions of Theorem 2.4 if $\mathscr{F}$ is transversely orientable.

If $\mathscr{F}$ is not transversely oriented let $\pi: \tilde{M} \rightarrow M$ be a 2 -fold cover such that $\tilde{\mathscr{F}}=\pi^{* \mathscr{F}}$ is transversely oriented. We show first that $\tilde{\mathscr{F}}$ has only one Novikov component by noting that we can lift either a closed transversal $w$ or $w^{2}$ from $M$ to $\tilde{M}$ and hence there exists a closed transversal through every leaf of $\tilde{M}$ and thus $\tilde{F}$ has only one Novikov component. Next transversely orient $\tilde{\mathscr{F}}$ and let $\tilde{f}=f \circ \pi$; 
then $S(\tilde{f})=\pi^{-1}(S \mid f)$ and the arc in $S(f)$ from $p$ to $q$ can be lifted to an arc in $S(\tilde{f})$ from $\tilde{p}$ to $\tilde{q}$ with $\tilde{p}$ a positive turning point of index $\lambda$ and $\tilde{q}$ a negative turning point of index $\lambda$. Let $\tilde{w}: I \rightarrow \tilde{M}$ be a positively oriented transversal from $\tilde{p}$ to $\tilde{q}$ such that $\tilde{w}(t)$ is an $\tilde{\mathscr{F}}$-regular point for $0<t<1$ and such that $\pi \circ \tilde{w}$ is a diffeomorphism-we use the fact that $n \geqslant 3$ here. Then there is a neighborhood $\tilde{U}$ of $\tilde{w}(I)$ such that $\pi \mid \tilde{U}$ is a diffeomorphism onto $U \subset M$. We have $f\left|U=\tilde{f} \circ \pi^{-1}\right| U$. Now let $\tilde{f}$ be the generic function of Theorem 2.4; $\overline{\tilde{f}}$ has no turning points in $\tilde{U}$ and $\tilde{\tilde{f}}|\tilde{M}-\tilde{U}=\tilde{f}| \tilde{M}-\tilde{U}$. We define $\bar{f}$ by $\bar{f}|M-U=f| M-U$ and $\bar{f}\left|U=\overline{\tilde{f}} \circ \pi^{-1}\right| U$. Note that the turning points $p$ and $q$ have been cancelled.

If $\mathscr{F}$ has only one component then Theorem 2.1 says that we can find a generic function $f:(M, \mathscr{F}) \rightarrow \mathbf{R}$ such that $S(f)$ is a disjoint union of closed transversals. In fact, one can do a bit better.

THEOREM 2.5. If $\mathscr{F}$ has only one Novikov component, then there is a generic function $g:(M, \mathscr{F}) \rightarrow \mathbf{R}$ such that $S(g)=S_{0} \cup S_{1}, \ldots, S_{n-1}$, where the $S_{i}$ are closed transversals and the leaf index of $S_{i}$ is $i$.

Sketch of Proof. One starts with an $f:(M, \mathscr{F}) \rightarrow \mathbf{R}$ having no turning points.

Let $\mathcal{O} \subset M-S(f)$. One then modifies $f$ to get a generic function $\tilde{f}$ such that $\tilde{f}|M-\mathcal{O} \equiv f| M-\mathcal{O}$ and with $S(\tilde{f})=S(f) \cup S$ where $S \subset \mathcal{O}$ and $S$ contains $(n-1)$ pairs of turning points at index $0,1, \ldots, n-2$. It is easier to introduce turning points than to cancel them and one can explicitly write down $\tilde{f} \mid \mathcal{O}$. Next, on each circle in $S(f)$ of leaf index $\lambda$ we introduce a pair of turning points of index $\lambda$ by slightly modifying $\tilde{f}$. We then cancel one of each pair with a turning point on $S$. The resulting function $h$ has $S(h)$ connected and $S(h)$ has $(n-1)$ pairs of turning points of index $0, \ldots, n-2$. Finally, cancelling all remaining turning points produces a function $g$ with $S(g)=S_{0} \cup \cdots \cup S_{n-1}$.

3. The two-dimensional cancellation theorem. The object of this section is to prove the following theorem.

THEOREM 3.1. Let $R^{2}$ be foliated by $x=$ constant and let $f: \mathbf{R}^{2} \rightarrow \mathbf{R}$ be generic. Let $w: I \rightarrow \mathbf{R}^{2}$ be a positively oriented transversal with $w(0)=p$ a positive turning point, $w(1)=q$ a negative turning point and $w(t)$ an $\mathscr{F}$-regular point for $0<t<1$. Then, given $\varepsilon>0$ there is an $\bar{f}: \mathbf{R}^{2} \rightarrow \mathbf{R}$ such that

(i) $\bar{f}=$ foutside of $N_{\varepsilon}(w(I))=\varepsilon$ neighborhood of $w(I)$,

(ii) $|\bar{f}-f|<\varepsilon$,

(iii) $\bar{f}$ has two fewer turning points than $f$ in $N_{\varepsilon}(w)$.

The proof of Theorem 3.1 occupies the remainder of this section. The plan is to alter $f$ as shown below in Figures 3 .

The key to the proof is the realization that cancellation of turning points is accomplished via the creation of critical points on the leaves. This is not very difficult, though the details are somewhat tedious. 

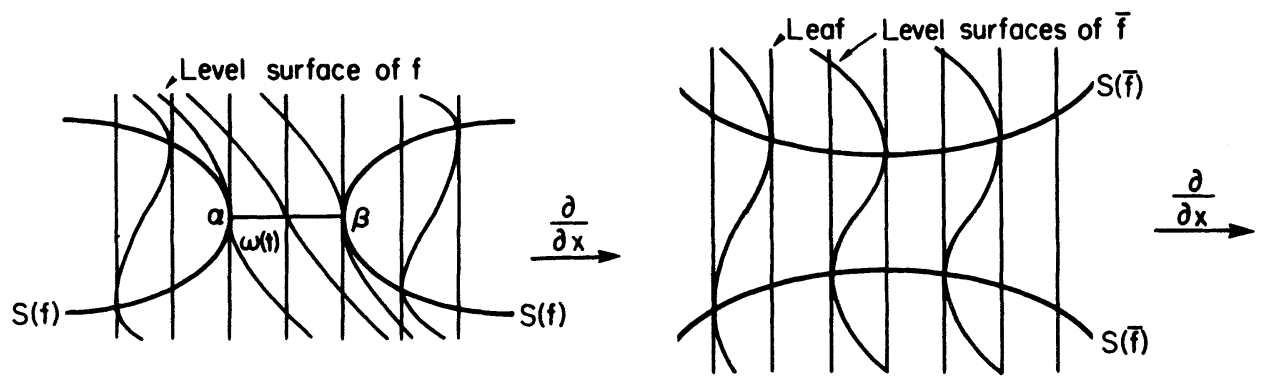

FIGURE 3. Before and after the cancellation of the turning points $\alpha$ and $\beta$

It is easy to change $f$ by precomposing with a leaf-preserving diffeomorphism to get $p=(0,0), q=(1,0)$, and $w(t)=(0, t)$. We will assume that this alteration has been accomplished. By sending $(x, y) \rightarrow(x,-y)$, if necessary, we can assume that $\partial f / \partial y>0$ on the open interval from $p$ to $q$. Hereafter, we will denote the open interval from $p$ to $q$ by $\widehat{p q}$ and the closed interval from $p$ to $q$ by $\overline{p q}$.

Proposition 3.2. Let $f$ be as above and let $\varepsilon>0$ be given. Then there is an $\tilde{f}$ : $\mathbf{R}^{2} \rightarrow \mathbf{R}^{1}$ such that

(i) $\tilde{\tilde{f}} \equiv$ foutside of $N_{\varepsilon}(\{p\} \cup\{q\})$,

(ii) $|\tilde{f}-f|<\varepsilon / 2$,

(iii) $\tilde{f}(x, y)=f(x, 0)+x y+y^{3} / 3$ in a neighborhood of $p$ and $\tilde{f}(x, y)=f(x, 0)-$ $(x-1) y+y^{3} / 3$ in a neighborhood of $q$.

Proof. See [F].

Proposition 3.3. Let $\tilde{f}$ be defined as above. Then there is a neighborhood $U$ of $\overline{p q}$ and a leaf-preserving diffeomorphism $\Phi: U \rightarrow \mathbf{R}^{2}$ such that

(i) $\Phi$ is the identity in a neighborhood of $\{p\} \cup\{q\}$.

(ii) $\Phi$ is the identity on $\overline{p q}$

(iii) $\tilde{f} \circ \Phi(x, y)=\tilde{f}(x, 0)+v(x) y+y^{3} / 3$ where

(iv) $v(x) \equiv x$ in a neighborhood of $p$ and $v(x) \equiv 1-x$ in a neighborhood of $q$.

PROOF. We seek a transformation of the form

$$
\Phi(x, y)(x,[1+\gamma(x, y)] y)
$$

where $\gamma(x, 0)=0$.

We write

$$
\begin{aligned}
\tilde{f}(x, y) & =\tilde{f}(x, 0)+\int_{0}^{1} \frac{\partial}{\partial t} \tilde{f}(x, t y) d t \\
& =\tilde{f}(x, 0)+y \tilde{f}_{1}(x, y) \\
& =\tilde{f}(x, 0)+y \tilde{f}_{1}(x, 0)+y^{2} \tilde{f}_{2}(x, y) .
\end{aligned}
$$

For our function $v(x)$, we choose $\tilde{f}_{1}(x, 0)=\partial \tilde{f} /\left.\partial y\right|_{(x, 0)}$. Then

$$
\tilde{f} \circ \Phi(x, y)=\tilde{\tilde{f}}(x, 0)+(1+\gamma) y v(x)+(1+\gamma)^{2} \tilde{\tilde{f}}_{2}(x, y) .
$$


To find the desired function $\gamma$, we must solve the equation

$\tilde{f}(x, 0)+(1+\gamma) y v(x)+(1+\gamma)^{2} y^{2} \tilde{f}_{2}(x,(1+\gamma) y)=\tilde{f}(x, 0)+v(x) y+y^{3} / 3$

for $\gamma$. That is, we must find $\gamma=\gamma(x, y)$ such that $\psi(x, y, \gamma) \equiv 0$ where

$$
\psi(x, y, \gamma)=\gamma v(x)+(1+\gamma)^{2} \tilde{f}_{2}(x,(1+\gamma) y) y-y^{2} / 3 .
$$

Note that $\psi(x, 0,0)=0$ and that $\partial \psi(x, 0, \gamma) / \partial \gamma=v(x)$. Therefore, there is a unique $\gamma=\gamma(x, y)$ with $\gamma(x, 0)=0$ solving the equation in some neighborhood $U$ of $\widehat{p q}$. Since $\gamma(x, y) \equiv 0$ is a solution in a neighborhood of $\{p\} \cup\{q\}, \gamma$ can be defined in a neighborhood of $\overline{p q}$. This completes the proof.

We will now assume that $\tilde{\tilde{f}}(x, y)$ has the form of $\tilde{\tilde{f}} \circ \Phi(x, y)$ above in $N_{\varepsilon}(w)$. Let $\delta>0$ be given. Let $\tilde{v}(x)$ be a function such that

(i) $\tilde{v}(x)=v(x)$ for $x \leqslant-\delta^{2}$ and for $x>1+\delta^{2}$.

(ii) $-\delta^{2}<\tilde{v}(x)<0$ for all $x \in\left(-\delta^{2}, 1+\delta^{2}\right)$.

See Figure 4. Let $\tilde{f}(x, y)=\tilde{f}(x, 0)+\tilde{v}(x) y+y^{3} / 3$.

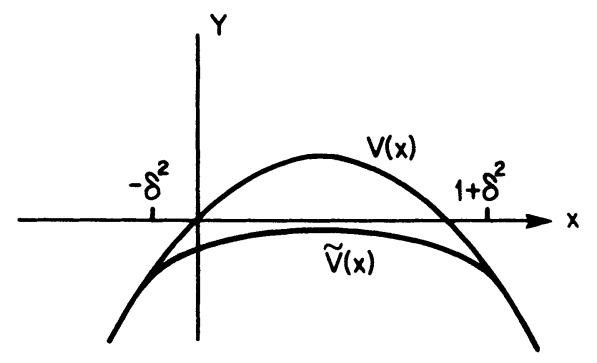

Figure 4

Proof (Theorem 3.1). Let $\rho=\rho(t)$ be a function such that

$$
\rho(t)= \begin{cases}1 & \text { for }|t|>3 \delta \\ 0 & \text { for }|t|<2 \delta\end{cases}
$$

and such that $\rho^{\prime}(t) \cdot t \geqslant 0$ for all $\tau$. We claim that for

$$
\delta<\min (\varepsilon / 3, \varepsilon / 2(v(x)+1), 1)
$$

the function $\bar{f}(x, y)=\rho(y) \tilde{\tilde{f}}(x, y)+(1-\rho(y)) \tilde{f}(x, y)$ satisfies the condition of Theorem 3.1.

Condition (i) is clear.

Condition (ii) follows easily from our choice of $\delta$ and the fact that $|\tilde{f}-f|=$ $y|v(x)-\tilde{v}(x)|$.

To see that condition (iii) is satisfied we compute as follows:

$$
\begin{aligned}
\tilde{f}(x, y) & =\tilde{\tilde{f}}(x, 0)+y^{3} / 3+\rho(y) v(x) y+(1-\rho(y)) \tilde{v}(x) y \\
& =\tilde{f}(x, 0)+y^{3} / 3+\rho(y) y[v(x)-\tilde{v}(x)]+\tilde{v}(x) y \\
& =\tilde{f}(x, 0)+\left[y^{3} / 3+\tilde{v}(x) y\right]+\rho(y) y[v(x)-\tilde{v}(x)] .
\end{aligned}
$$

Then

$$
\frac{\partial \bar{f}}{\partial y}=\left[y^{2}+\tilde{v}(x)\right]+\rho(y)[v(x)-\tilde{v}(x)]+\rho^{\prime}(y) y[v(x)-\tilde{v}(x)] .
$$


For $|y|>\delta$ and $-\delta^{2}<x<1+\delta^{2}$, each of the three terms on the right are nonnegative and the first term is positive. Therefore, there are no pseudocritical points of $\bar{f}$ in this area. For $|y|<2 \delta$ or $x<-\delta^{2}$ or $x>1+\delta^{2}$, we have $\rho(y) \equiv 0$ and $\tilde{f}(x, y)=\tilde{f}(x, y)$, which is easily seen to have two regular pseudocritical points on each segment $x=$ constant, $-3 \delta<y<3 \delta$ namely $y= \pm \sqrt{\tilde{v}(x)}$. Thus the singular set is not tangent to the leaves in this region and this completes the proof of Theorem 3.1 .

4. The cancellation theorem. The object of this section is to prove Theorem 2.4. Since Theorem 2.4 concerns a modification of a function in a neighborhood of a transverse arc, $w(I)$, we may, by taking a tubular neighborhood of $w(I)$, reduce the proof of the proof of Proposition 4.1.

Proposition 4.1. Let $\mathbf{R}^{n}$ be foliated by $x=$ constant. Let $f: \mathbf{R}^{n} \rightarrow \mathbf{R}$ be a generic function such that $f$ has a positive turning point of index $\lambda$ at $p=(0, \ldots, 0)$ and a negative turning point of index $\lambda$ at $q=(1,0, \ldots, 0)$. Suppose the open arc $\overrightarrow{p q}$ does not meet the singular set, $S(f)$. Then given any open neighborhood $\mathcal{O}$ of the closed arc $\overline{p q}$ there is a smooth generic function $\bar{f}: \mathbf{R}^{n} \rightarrow \mathbf{R}$ such that

(i) $f \equiv \bar{f}$ outside of $\mathcal{O}$

(ii) $\bar{f}$ has two fewer turning points than $f$.

The proof of Proposition 4.1 would be trivial if $f(x, y, z)$ were of the form $h(x, y)+\sum_{i=1}^{n-2} \pm z_{i}^{2}$ using the two-dimensional result, Theorem 3.1. In fact, the weaker splitting theorem below is enough.

Proposition 4.2. If $f: \mathbf{R}^{n} \rightarrow \mathbf{R}$ is as in Proposition 4.1, then there is an open neighborhood of $\overline{p q}, \mathcal{O}_{1} \subset \mathcal{O}$, and a diffeomorphism $\Phi: \mathcal{O}_{1} \rightarrow \mathcal{O}$ such that

(i) $f \circ \Phi(x, y, Z)=h(x, y)+\sum_{i=1}^{n-2} z_{i} f_{i}(x, y, Z)$,

(ii) $f_{i}(x, 0,0)=0$,

(iii) $f_{i}(x, y, Z)=\varepsilon_{i} z_{i}$ is a small neighborhood $U$ of $\{p\} \cup\{q\}$ where $\varepsilon_{i}=-1$ if $0 \leqslant i \leqslant \lambda$ and $\varepsilon_{i}=+1$ if $\lambda<i$,

(iv) $\Phi(x, y, Z)=\left(x, \Psi_{x}(y, Z)\right)$,

(v) $\Phi(p)=p, \Phi(q)=q$.

REMARK. Subscripts do not indicate partial derivatives.

We now show that Proposition 4.2 implies Proposition 4.1.

Proof of Proposition 4.1. Let $g=f \circ \Phi$. We will modify $g$ (in two steps) so that $\overline{\bar{g}}$ has no turning points and then set

$$
\bar{f}(x, y, Z)= \begin{cases}\overline{\bar{g}} \circ \Phi^{-1}(x, y, Z) & \text { if }(x, y, Z) \in \Phi\left(\mathcal{O}_{1}\right), \\ f(x, y, Z) & \text { if }(x, y, Z) \notin \Phi\left(\mathcal{O}_{1}\right) .\end{cases}
$$

Clearly $\bar{f}$ will then satisfy the conditions of Proposition 4.1.

Let $\bar{\rho}: \mathbf{R} \rightarrow \mathbf{R}$ satisfy

(i) $\bar{\rho}(t)=0$ for $t \geqslant 1$,

(ii) $\bar{\rho}(t)=1$ for $t \leqslant \frac{1}{2}$,

(iii) $\bar{\rho}^{\prime}(t) \leqslant 0$. 
By (i) of Proposition 4.2 we have, for $(x, y, Z) \in \mathcal{O}_{1}$,

$$
g(x, y, z)=h(x, y)+\sum_{i=1}^{n-2} z_{i} f_{i}(x, y, Z) \text {. }
$$

Set

$$
\bar{g}(x, y, Z)=g(x, y, Z)+\bar{\rho}\left(K\left(y^{2}+|Z|^{2}\right)\right)\left(\sum_{i=1}^{n-2} z_{i}\left(\varepsilon_{i} z_{i}-f_{i}(x, y, Z)\right)\right)
$$

where $K$ is a positive constant to be chosen.

Note that $\bar{g}=g$ if $\left(y^{2}+|Z|^{2}\right) \geqslant 1 / K$, on $\overline{p q}$, and also in a neighborhood $U$ of $\{p\} \cup\{q\}$ by (ii). Choose $K$ large enough so that $\left\{\left.(x, y, Z)\left|0 \leqslant x \leqslant 1, y^{2}+\right| Z\right|^{2}\right.$ $\leqslant 1 / K\} \subset \mathcal{O}_{1}$ and $S(\bar{g})=S(g)$. This is possible since, first of all, $g|U=\bar{g}| U$ so $S(\bar{g} \mid U)=S(g \mid U)$ and, secondly, $g-\bar{g}$ vanishes to second order on $\overline{p q}$ and therefore $d g$ and $d \bar{g}$ are close near $\overline{p q}$. But $d(g \mid L) \neq 0$ on $X-U$ so, for $K$ sufficiently large, $d(\bar{g} \mid L) \neq 0$ on $X-U$ where $X$ is a compact set containing support $(g-\bar{g})$. Thus $S(\bar{g})=S(g)$. Now, choose an open neighborhood $\mathcal{O}_{2}$ of $\overline{p w}$ such that $y^{2}+|z|^{2}<1 / 2 K$. Then $\bar{g} \mid \mathcal{O}_{2}$ has the particularly simple form

$$
\bar{g}(x, y, z)=h(x, y)+\sum_{i=1}^{n-2} \varepsilon_{i} z_{i}^{2} .
$$

The function $h(x, y)$ is a generic function on an open set in $\mathbf{R}^{2}$ with a positive turning point at $p$ and a negative turning point at $\varepsilon$. By Theorem 3 we can find $\bar{h}$ : $\mathcal{O}_{1} \rightarrow \mathbf{R}$ such that

(i) $\bar{h} \equiv h$ outside $\mathcal{O}_{2}$,

(ii) $\sup |\bar{h}-h|<\eta$,

(iii) $\bar{h}$ has no turning points in $\mathcal{O}_{2}$.

Finally, define $\overline{\bar{g}}$ by

$$
\overline{\bar{g}}(x, y, Z)=\bar{g}(x, y, Z)+\bar{\rho}\left(2 K|Z|^{2}\right)(\bar{h}(x, y)-h(x, y)) .
$$

Note that, for $|Z|^{2}<1 / 4 K$,

$$
\overline{\bar{g}}(x, y, Z)=\bar{h}(x, y)+\sum_{i=1}^{n-2} \varepsilon_{i} z_{i}^{2}
$$

thus $\overline{\bar{g}}$ has no turning points in this region. Similarly, if $|Z|^{2}>1 / 2 K, \overline{\bar{g}}=\bar{g}$ and hence there are no turning points in this region either. Writing

$$
\overline{\bar{g}}(x, y, Z)=\sum_{i=1}^{n-2} \varepsilon_{i} z_{i}^{2}+\bar{h}(x, y)+\bar{\rho}\left(2 K|Z|^{2}\right)(h(x, y)-\bar{h}(x, y))
$$

we compute

$$
\begin{aligned}
\frac{\partial \overline{\bar{g}}}{\partial z_{i}} & =2 \varepsilon_{i} z_{i}+4 K z_{i} \bar{\rho}^{\prime}\left(2 K|Z|^{2}\right)(h(x, y)-\bar{h}(x, y)) \\
& =z_{i}\left(2 \varepsilon_{i}+4 K \bar{\rho}^{\prime}\left(2 K|Z|^{2}\right)(h(x, y)-\bar{h}(x, y))\right) .
\end{aligned}
$$


Since $\left|2 \varepsilon_{i}\right|=2$ and $\bar{\rho}^{\prime}$ is bounded we can choose $\eta>0$ sufficiently small so that $\partial \overline{\bar{g}} / \partial z_{i}=0$ iff $z_{i}=0$. Thus all the pseudocritical points of $\overline{\bar{g}}$ are contained in the region $|Z|^{2}<1 / 4 K$ and hence $\overline{\bar{g}}$ has no turning points.

Finally, we turn to the proof of Proposition 4.2.

Proof of Proposition 4.2. By Theorem 1.2, there is a leaf-preserving coordinate change $\Phi_{p}: U_{0} \rightarrow \mathbf{R}^{n}$ whose $U_{0}$ is an open set about the origin in $\mathbf{R}^{n}$ such that $\Phi_{p}(0)=p$,

$$
f \circ \Phi_{p}(x, y, Z)=x+x y+\psi(y)+Q(Z)=h(x, y)+Q(Z)
$$

where $Q(Z)$ is a nondegenerate quadratic form of index $\lambda$. Furthermore, by a linear change of coordinates we may diagonalize $Q$ getting

$$
f \circ \tilde{\Phi}_{p}(x, y, z)=h(x, y)+\sum_{i=1}^{n-2} \varepsilon_{i} z_{i}^{2}
$$

where $\varepsilon_{i}=-1$ for $0<i \leqslant \lambda$ and $\varepsilon_{i}= \pm 1$ for $i>\lambda$. Similarly, we may find a leaf-preserving coordinate change $\Phi_{q}: U_{1} \rightarrow \mathbf{R}^{n}$ where $U_{1}$ is an open set about $q=(1,0, \ldots, 0) \in \mathbf{R}^{n}, U_{0} \cap U_{1}=\varnothing$ and with

$$
f \circ \Phi_{q}(x, y, Z)=h(x, y)+\sum_{i=1}^{n-2} \varepsilon_{i} z_{i}^{2}
$$

as before. Putting these together yields a leaf-preserving diffeomorphism $\Phi_{1}: U \rightarrow \mathbf{R}^{n}$ where $U$ is a neighborhood of $\{p\} \cup\{q\}$ and

$$
f \circ \Phi_{1}(x, y, z)=h(x, y)+\sum_{i=1}^{n-2} \varepsilon_{i} z_{i}^{2} .
$$

Furthermore, we may assume $\Phi_{1}$ is orientation-preserving on both components of $U$. If not, we may compose $\Phi_{1}$ with reflection in the $z_{1}=0$ plane to get an orientation-preserving diffeomorphism with the same properties. Let us write $\Phi_{1}(x, y, Z)=\left(x, \Psi_{x}(y, Z)\right)$.

The required diffeomorphism $\Phi$ must be defined on a neighborhood of $\overline{p q}$. Choose $\delta>0$ such that $V \subset U$ where

$$
V=\left\{(x, y, Z) \mid-\delta<x<3 \delta \text { or } 1-3 \delta<x<1+\delta \text { and } y^{2}+|Z|^{2}<\delta\right\} ;
$$

we will construct a new diffeomorphism $\Phi_{2}: V \rightarrow \mathbf{R}^{n}$ such that

(i) $\Phi_{2}(x, y, Z)=\Phi_{1}(x, y, Z)$ if $|x| \leqslant \delta$ or $|x-1| \leqslant \delta$,

(ii) $\Phi_{2}(x, y, Z)=\left(x, L_{x}(y, Z)\right)$ if $2 \delta<x<3 \delta$ where $L_{x}: \mathbf{R}^{n-1} \rightarrow \mathbf{R}^{n-1}$ is a linear map for each $x$.

Define $\Phi_{2}$ as follows:

$$
\Phi_{2}(x, y, Z)=\left\{\begin{array}{r}
\left(x, \frac{1}{\bar{\rho}(x / 2 \delta)} \Psi_{x}\left(\bar{\rho}\left(\frac{x}{2 \delta}\right) y, \bar{\rho}\left(\frac{x}{2 \delta}\right) Z\right)\right) \text { if } x<2 \delta, \\
\left(x, d \Psi_{x}(y, Z)\right) \text { if } 2 \delta \leqslant x<3 \delta \text { or } 1-3 \delta<x \leqslant 1-2 \delta, \\
\left(x, \frac{1}{\bar{\rho}((1-x) / 2 \delta)} \Psi_{x}\left(\bar{\rho}\left(\frac{1-x}{2 \delta}\right) y, \bar{\rho}\left(\frac{1-x}{2 \delta}\right) Z\right)\right) \\
\text { if } 1-2 \delta<x,
\end{array}\right.
$$


where $\bar{\rho}$ is the bump function used in the proof of 4.1. It is well known that this "Alexander isotopy" defines a smooth diffeomorphism $\Phi_{2}$.

Next let $\gamma:[2 \delta, 1-2 \delta] \rightarrow \operatorname{GL}(n-1, \mathbf{R})$ be a smooth map satisfying $\gamma(x)=d \Psi_{x}$ for $2 \delta<x<2.5 x$ and $1-2.5 \delta \leqslant x \leqslant 1-2 \delta$. We may find such a map since $d \Psi_{x}$ is orientation-preserving for all $x$.

Let $V=\left\{(x, y, Z) \mid-\delta<x<1+\delta\right.$ and $\left.y^{2}+|Z|^{2}<\delta\right\}$ and define $\Phi_{3}: V \rightarrow \mathbf{R}^{n}$ by

$$
\Phi_{3}(x, y, Z)= \begin{cases}\Phi_{2}(x, y, Z) & \text { if }-\delta<x \leqslant 2.5 \delta \text { or } 1-2.5 \delta \leqslant x<1+\delta, \\ (x, \gamma(x)(y, Z)) & \text { if } 2.5 \delta \leqslant x \leqslant 1-2.5 \delta .\end{cases}
$$

Note that the diffeomorphism $\Phi_{3}$ is defined on a neighborhood of $\overline{p q}, \Phi_{3}(p)=p$, and $\Phi_{3}(q)=q$, and $\Phi_{3}(x, y, Z)=\left(x, \Psi_{x}(y, Z)\right)$. In fact, $\Phi_{3}$ satisfies conditions (i), (iii), (iv) and (v) of Proposition 4.2. To satisfy (ii), however, we need $\left(\partial / \partial z_{i}\right) f \circ \Phi(x, 0,0)=0$ or that $\nabla(f \circ \Phi \mid L(x))$ is a multiple of $\partial / \partial y$. Recall that the open arc $\widehat{p q}$ does not meet the singular set $S(f)$, therefore, for $0<x<1$,

$$
s(x)=\nabla\left(f \circ \Phi_{3} \mid L(x)\right)(x, 0,0) \neq 0 .
$$

Let $\bar{s}(x)=s(x) /\|s(x)\| \in S^{n-2}$. Note that $\bar{s}(x)=\partial / \partial y=(1,0, \ldots, 0)$ for $x$ near 0 or 1 i.e. near $p$ or $q$. Extend $\bar{s}$ to $\mathbf{R}$ by $\bar{s}(x)=\partial / \partial y$ for $x \notin(0,1)$.

Let $\pi: \mathrm{SO}(n-1) \rightarrow S^{n-2}$ be the standard fibration; $\pi(A)=$ first column of the matrix $A$. Let $s: \mathbf{R} \rightarrow \mathrm{SO}(n-1)$ be a smooth lift of $\bar{s}$ such that $s(x)=$ id for $x \notin(0,1)$. Define a leaf-preserving diffeomorphism of $\mathbf{R}^{n}, \Phi_{4}: \mathbf{R}^{n} \rightarrow \mathbf{R}^{n}$ by $\Phi_{4}(x, y, Z)=(x, s(x)(y, Z))$. We claim that $\Phi=\Phi_{3} \circ \Phi_{4}$ is the desired diffeomorphism.

Since both $\Phi_{3}$ and $\Phi_{4}$ are leaf preserving so is $\Phi$; similarly, $\Phi_{3}(p)=p, \Phi_{4}(p)=p$ so $\Phi(p)=p$ and also $\Phi(q)=q$. Condition (i) is free:

$$
\begin{aligned}
f \circ \Phi(x, y, Z) & =f \circ \Phi(x, y, 0)+\int_{0}^{1} \frac{\partial}{\partial t} f \circ \Phi(x, y, t Z) d t \\
& =h(x, y)+\int_{0}^{1} \sum_{i=1}^{n-2} z_{i} \frac{\partial}{\partial z_{i}} f \circ \Phi(x, y, t Z) d t \\
& =h(x, y)+\sum_{i=1}^{n-2} z_{i} \int_{0}^{1} \frac{\partial}{\partial z_{i}} f \circ \Phi(x, y, t Z) d t \\
& =h(x, y)+\sum_{i=1}^{n-2} z_{i} f_{i}(x, y, Z)
\end{aligned}
$$

where

$$
f_{i}(x, y, Z)=\int_{0}^{1} \frac{\partial}{\partial z_{i}}(f \circ \Phi(x, y, t Z)) d t .
$$

In a small neighborhood of $\{p\} \cup\{q\}$ we have $\Phi_{4}=\mathrm{id}$ and $\Phi_{3}=\Phi_{1}$ so

$$
f \circ \Phi(x, y, z)=h(x, y)+\sum_{i=1}^{n-2} \varepsilon_{i} z_{i}^{2}
$$


and hence,

$$
f_{i}(x, y, Z)=\int_{0}^{1} 2 \varepsilon_{i} z_{i} t d t=\varepsilon_{i} z_{i}
$$

as required by (iii). Finally,

$$
f_{i}(x, 0,0)=\int_{0}^{1} \frac{\partial}{\partial z_{i}} f \circ \Phi(x, 0,0) d t=\frac{\partial}{\partial z_{i}} f \circ \Phi(x, 0,0) .
$$

But $\Phi_{4}$ was chosen so that $\nabla f \circ \Phi(x, 0,0)$ would be a positive multiple of $\partial / \partial y$, i.e.

$$
\frac{\partial}{\partial z_{i}} f \circ \Phi(x, 0,0)=0=f_{i}(x, 0,0) \text {. }
$$

\section{REFERENCES}

[F] S. Ferry, Codimension one Morse theory, Thesis, Univ. of Michigan, 1973.

[H] A. Haefliger, Quelques remarques sur les applications différentiables d'une surface dans le plan, Ann. Inst. Fourier (Grenoble) 10 (1960), 47-60.

[H-W] A. Hatcher and J. Wagoner, Pseudo-isotopies of compact manifolds, Asterisque, No. 6, 1973.

$\left[\mathbf{L}_{1}\right]$ H. I. Levine, Elimination of cusps, Topology 3 (1965), 263-296.

$\left[\mathbf{L}_{2}\right]$ _ 1959.

[M] J. W. Milnor, Morse theory, Princeton Univ. Press, Princeton, N. J., 1963.

[N] S. P. Novikov, Topology of foliations, Trans. Moscow Math. Soc. 14 (1965), 268-304.

$\left[T_{1}\right]$ R. Thom, Les singularitiés des applications différentiables, Ann. Inst. Fourier (Grenoble) 6 (1955), 43-87.

$\left[\mathbf{T}_{2}\right] \ldots$, Généralisation de la théorie de Morse aux variétés feuilletées, Ann. Inst. Fourier (Grenoble) 14 (1964), 173-189.

[Wa] Y. H. Wan, Morse theory for two functions, Topology 14 (1975), 217-228.

[W] H. Whitney, On singularities of mappings of euclidean spaces. I: Mappings of the plane into the plane, Ann. of Math. (2) 62 (1955), 374-410.

Department of Mathematics, University of Kentucky, Lexington, Kentucky 40506

Department of Mathematics, University of Michigan, Ann Arbor, Michigan 48109 